\title{
Numerical Investigation of Modal Parameter Statistics of Random Rods
}

\author{
Carlos H. Gomes and Samir N. Y. Gerges \\ Department of Mechanical Engineering, Federal Univ. of Santa Catarina, University Campus, Trindade, 88040- \\ 900, Florianpolis-SC, Brazil
}

\author{
Jorge P. Arenas \\ Institute of Acoustics, Univ. Austral de Chile, PO Box 567, Valdivia, Chile
}

\begin{abstract}
(Received 10 June 2013; accepted 6 June 2014)
In this article, the ensemble statistics of the modal parameters of longitudinal rods with non-deterministic parameters were investigated through the statistical results of metric functions of the Random Matrix Theory (RMT). The levels of agreement with the Poisson and Gaussian Orthogonal Ensemble (GOE) models were evaluated for natural frequencies and corresponding mode shapes. The mean and relative variance values of kinetic energy density responses were calculated and compared to analytical predictions based on the Poisson and GOE models. The main effects on the energy response statistics due to incomplete establishment of GOE (or Poisson) statistics were systematically assessed for each modal parameter considering the single point-loading and spatially-averaged excitation. The numerical results also allowed the identification of the possible impact on the performance of analytical predictions of the relative variance when the modal parameter statistics do not conform completely to the GOE (or Poisson) model. An improved understanding was obtained regarding the relationship between the modal parameter statistics expected for one-dimensional engineering systems and those described by the GOE and Poisson models.
\end{abstract}

\section{INTRODUCTION}

The response of a complex engineering system is strongly dependent upon the frequency of excitation. In the lowfrequency range, only the first modes of an engineering system are excited, and thus the dynamical response can be predicted with good accuracy by using a well-established deterministic method of analysis, ${ }^{1}$ for example, the Finite Element Method (FEM). ${ }^{2}$ In medium to high-frequency ranges, many hundreds of modes are excited, and it becomes extremely difficult to predict the precise response of a dynamical system since the higher-order modes are very sensitive to small variations introduced during the manufacturing and assembly processes, leading to a significant random spread for the energy response. ${ }^{1,3}$ Indeed, the large variability of the energy responses across the ensemble, beyond the first modes, is detrimental to engineering decisions during several steps of the design process of a certain mechanical component as well as in the certification process of the final product.

In order to overcome these engineering difficulties, the midand high-frequency dynamic behaviors of engineering systems with non-deterministic parameters are usually assessed through Statistical Energy Analysis (SEA), which is able to predict accurately the expected mean value of energy responses across an ensemble composed of nominally identical systems. ${ }^{4}$ More recently, Statistical modal Energy distribution Analysis (SmEdA) has been used as an alternative to SEA for describing subsystems with low modal overlap. .,6 $^{5}$

In recent decades, considerable interest has been focused on the possible extension of SEA models to confidently predict the relative variance of energy responses. The pioneering works were carried out in the field of room acoustics using the random point process approach ${ }^{7}$ where the natural frequencies are considered as random variables on the frequency axis so that the well-established statistical models of the Random Matrix Theory $(\mathrm{RMT})^{8}$ can be adopted to model the modal parameter statistics in order to derive analytical predictions for the statistical moments of energy responses.

Lyon $^{7}$ adopted the Poisson model to describe the natural frequency statistics of simple systems (such as, a simplysupported rectangular plate or a rectangular acoustic room) and thus derived analytical predictions for the variance of the energy responses of a single subsystem subjected to single-point loading. Although the analytical predictions based on the Poisson model are very convenient from the analytical point view, their application is restricted to single systems with several symmetries or built-up systems with several non-interacting components. ${ }^{9}$ For practical engineering systems, the structural uncertainties lead to the introduction of spectral correlations between the natural frequencies, and thus substantial deviations from perfect Poisson eigenvalues are clearly established, invaliding the application of Poisson predictions in the midand high-frequency ranges. ${ }^{10}$ Additionally, some relevant results obtained in the area of physics with systems of several natures have shown that, except for the systems with regular geometry, the statistics of natural frequencies are very similar to those described by the eigenvalues of a special type of large random symmetric matrix called the Gaussian Orthogonal Ensemble (GOE) from RMT. ${ }^{11}$ Supported by this practical evidence, several efforts have been made to derive the analytical predictions for SEA variance based on the GOE model. ${ }^{12-14}$ Even though the GOE predictions provide a substantial im- 